\title{
Identificación de especies ecológicamente relevantes para la Evaluación de Riesgo Ecológico: Una propuesta desde la ecología teórica
}

\author{
Identification of ecologically relevant species for Ecological Risk Assessment: A proposal \\ from theoretical ecology
}

\section{RODRIGO RAMOS-JILIBERTO ${ }^{1,2}{ }^{*}$, PASQUINELL URBANI ${ }^{1}$, LESLIE GARAY-NARVÁEZ1 ${ }^{1}$, PABLO RAZETO-BARRY²,3, FRANCISCO ENCINA-MONTOYA ${ }^{4} \&$ MATÍAS H. MEDINA ${ }^{5}$
${ }^{1}$ Centro Nacional del Medio Ambiente. Fundación de la Universidad de Chile. Av. Larraín 9975 La Reina, Santiago, Chile ${ }^{2}$ Instituto de Filosofía y Ciencias de la Complejidad, IFICC. Los Alerces 3024, Santiago, Chile
${ }^{3}$ Universidad Diego Portales, Vicerrectoría Académica, Manuel Rodríguez Sur 415, Santiago ${ }^{4}$ Escuela de Ciencias Ambientales. Facultad de Recursos Naturales, Universidad Católica de Temuco, Manuel Montt 56, Temuco, Chile
${ }^{5}$ Instituto Tecnológico del Salmón, INTESAL de SalmonChile, Juan Soler Manfredini 41 Of. 1802, Puerto Montt, Chile \\ * Autor correspondiente: ramos.jiliberto@gmail.com}

\section{RESUMEN}

En base a avances recientes en teoría de redes ecológicas, se presenta un marco conceptual ad hoc y una propuesta metodológica para la evaluación de la relevancia ecológica de las especies de una comunidad, orientada a la Evaluación de Riesgo Ecológico de sistemas acuáticos en Chile. El procedimiento es de carácter teórico y se basa en la calidad de la información disponible, es decir no requiere de evaluaciones experimentales ni observacionales de campo. Dado el estado de conocimiento actual y esperado a mediano plazo acerca de la estructura de las comunidades acuáticas locales, el método propuesto está enfocado a su aplicación en comunidades cuyo nivel de conocimiento es bajo o medio. La propuesta utiliza criterios cualitativos e índices cuantitativos (siete índices topológicos y un índice basado en modelación cualitativa) para la identificación de especies ecológicamente relevantes que representen al menos tres categorías tróficas de la comunidad.

Palabras clave: análisis de ciclos, especies clave, índices de centralidad, redes ecológicas, topología.

\section{ABSTRACT}

Based on recent advances in ecological network theory, we present an ad hoc conceptual framework and a methodological proposal for the evaluation of the ecological relevance of species within a community, oriented towards the Ecological Risk Assessment in Chilean aquatic systems. The procedure is theoretical and based on the quality of available information, i.e. it does not require experimental tests or field observation. Given the current and mediumterm expected state of knowledge about the structure of local aquatic communities, the proposed method is focused towards its application to communities whose level of knowledge is low or medium. The proposal uses qualitative criteria and quantitative indices (seven topological indices and one index based on qualitative modeling) for the identification of ecologically relevant species that represent at least three trophic categories within the community.

Key words: centrality indices, ecological networks, keystone species, loop analysis, topology.

\section{INTRODUCCIÓN}

La comprensión, predicción y retrodicción de los efectos ejercidos por factores naturales o antropogénicos sobre la estructura y función de los organismos, poblaciones y comunidades es una meta fundamental de la investigación ecológica. Sin embargo, los avances provenientes de la investigación ecológica no son ágilmente incorporados en las decisiones de manejo debido a una multiplicidad de factores sociales y políticos (Simonetti 2011). Hoy existen iniciativas que buscan introducir en la regulación ambiental chilena procedimientos científico-técnicos que permitan anticipar con mayor certidumbre la potencial ocurrencia de efectos adversos en las comunidades naturales como producto de la actividad humana. En este 
sentido, la Evaluación de Riesgo Ecológico (ERE) es uno de los instrumentos que están siendo discutidos actualmente.

La ERE es un proceso a través del cual se pretende estimar la probabilidad de que ocurran en el futuro, o estén ocurriendo en el presente, efectos ecológicos adversos en un ecosistema focal como resultado de la exposición a uno o más agentes estresantes liberados al ambiente por una actividad antrópica (USEPA 1992, Encina \& Díaz 2001). Las EREs pueden ser utilizadas para predecir la probabilidad de efectos adversos futuros (ERE prospectivo), como también para retrodecir las causas pasadas de efectos observados en el presente (ERE retrospectivo). De acuerdo a la U.S.E.P.A., el proceso de una ERE puede dividirse en tres fases (USEPA 1998): (i) Formulación del problema, en la cual se define el propósito particular de la evaluación y se diseña un plan de acción; (ii) análisis, el núcleo del ERE; y (iii) caracterización del riesgo, fase en la cual se integran los resultados y se determina una medida del riesgo. La fase de análisis, que es en la cual se enfoca este trabajo, se conforma de dos componentes: (a) Caracterización de la exposición, en que se estima la probabilidad de ocurrencia de estresantes en el ambiente objetivo y (b) Caracterización de efectos ecológicos en que, dada la exposición a un agente estresante, se estima la potencialidad y tipo de efectos esperados en el sistema ecológico objetivo. En este componente se aplican herramientas ecotoxicológicas para determinar la sensibilidad de las especies-objetivo cuyo valor se considera indicativo de la tolerancia de la comunidad completa (Fig. 1). Sin embargo, la selección de especies-objetivo se ha basado en criterios logísticos más que sistémicos. Según Van Leeuwen \& Hermens (1995) y Rand et al. (1995), las especies escogidas deben ser (i) fáciles de recolectar, (ii) abundantes y accesibles, (iii) fáciles de manipular y mantener en laboratorio y (iv) de ciclo de vida corto. Estos autores además agregan que las pruebas de toxicidad debieran privilegiar aquellas cuya sensibilidad a diversos agentes estresantes es mayor y para las cuales se tiene mayor información sobre su biología. Por último, estos autores destacan la necesidad que las especies escogidas sean representativas de la ruta de exposición, y que estas posean una importancia

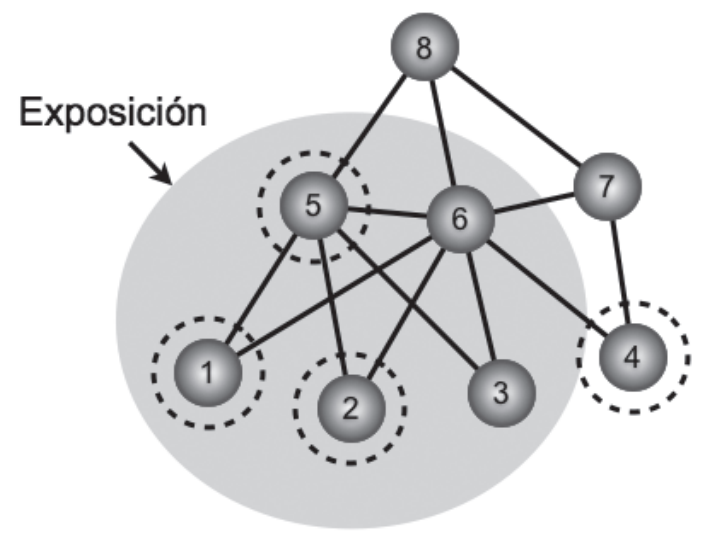

Fig. 1: Representación gráfica de una comunidad sujeto de ERE y conceptos asociados. Nodos y líneas rectas representan especies e interacciones. El área sombreada representa la zona de exposición a un estresante k. Especies 1, 2, 4 y 5, circundadas por círculos segmentados, representan especies más sensibles a $\mathrm{k}$. Las especies 5 y 6 poseen la mayor relevancia ecológica, atribuida a razones topológicas. Especies 1, 2 y 5 son las más susceptibles, puesto que presentan alta exposición y mayor sensibilidad a $\mathrm{k}$. La tolerancia de la especie 5 indica en mejor medida el riesgo de la comunidad frente a $\mathrm{k}$, puesto que (a) presenta alta susceptibilidad a $\mathrm{k}$, (b) presenta alta relevancia ecológica dentro de la comunidad.

Graphic representation of a community subjected to ecological risk assessment, and associated concepts. Nodes and straight lines represent species and interactions. Shaded area represents zone exposed to a stressor $\mathrm{k}$. Species 1, 2, 4 y 5 , surrounded by dashed circles, represent species most sensitive to $\mathrm{k}$. Species 5 and 6 have the highest ecological relevance. Species 1, 2 and 5 are more susceptible, since they present a high exposure and high sensitivity to $\mathrm{k}$. Tolerance of 5 indicates in a better way the community risk facing $\mathrm{k}$, since (a) it has a high susceptibility to $\mathrm{k}$, and (b) it has a high ecological relevance within the community.

(relevancia) recreacional, económica o ecológica. En este trabajo planteamos que los criterios logísticos y de sensibilidad a estresantes no deben ser el primer filtro para la elección de especies-objetivo sino que estos deben ser aplicados al subconjunto de especies identificadas como ecológicamente relevantes. Lo anterior, dado que (a) los criterios logísticos no guardan necesaria relación con las propiedades del sistema ecológico, (b) solo se conoce la sensibilidad de un número reducido de especies a un número reducido de estresantes y (c) conjeturamos que es posible estimar la relevancia ecológica de las especies de forma rápida y sencilla, en base a la información disponible del sistema en estudio. 
Sostenemos además que la identificación de las especies ecológicamente relevantes debe realizarse utilizando procedimientos científicos que impliquen la mayor objetividad posible. Este tema ha sido abordado como parte del proceso de discusión en que se encuentra la incorporación de la ERE en la legislación ambiental chilena, en que se espera mejorar la calidad de dicho proceso mediante la utilización de especies ecológicamente relevantes para cada ecosistema en que se desarrolle una ERE en asociación con la potencial liberación de agentes estresantes.

Por lo anterior resulta crucial para la gestión de los sistemas ecológicos a través de ERE, contar con procedimientos que permitan identificar correctamente las especies con mayor relevancia ecológica dentro de una comunidad. Frecuentemente, esta selección descansa en la opinión experta, en que especialistas con experiencia en el ecosistema focal indican en base a su conocimiento qué especies consideran relevantes. Sin embargo, el criterio experto como único instrumento de valoración de la relevancia ecológica de las especies presenta serios inconvenientes. Primero, es subjetivo y presenta un alto riesgo de estar sesgado en favor de los organismos preferidos del especialista. Por otro lado, el criterio experto requiere de importante conocimiento empírico acumulado acerca del sistema focal. Con ello, y ante el hecho que existen muy pocos ecosistemas en Chile y la Región que puedan considerarse bien estudiados, el criterio experto difícilmente otorgará valor diferencial para la mismas especies en ambientes diferentes, ignorando el contexto ecológico en que estas se encuentran. Esto sugiere la conveniencia de apoyarse en herramientas cuantitativas para la estimación de la relevancia ecológica de las especies de cualquier comunidad de interés. Estas herramientas deberían requerir del mínimo de información empírica como entrada, a fin que su aplicación sea factible en el corto plazo.

El objetivo de este estudio es presentar una propuesta metodológica para definir especies de relevancia ecológica en los sistemas hídricos de Chile, que estén o puedan estar siendo afectados por sustancias químicas tóxicas liberadas al ambiente como sub-producto de actividades humanas. Para este fin, en este trabajo (a) se desarrolló un marco conceptual para la correcta interpretación de la relevancia ecológica en el marco de Evaluación de Riesgo Ecológico, (b) se realizó una revisión y selección de herramientas cualitativas y cuantitativas, desarrolladas en la investigación ecológica y ecotoxicológica, que presenten potencial utilidad como instrumento de identificación de las especies ecológicamente relevantes (EER) en sistemas hídricos locales y (c) se propone un procedimiento que integra la información parcial de las métricas seleccionadas a fin de otorgar un valor de relevancia ecológica a cada especie de un sistema focal definido.

\section{RIESGO ECOLÓGICO}

En una ERE se define previamente tanto el ecosistema focal para los propósitos de manejo, como la propiedad de la comunidad que se requiere proteger. A modo de ejemplo, podría desearse proteger una especie carismática particular dentro de la comunidad (e.g., una especie de cisne), un gremio de interés (e.g., el conjunto de corales, o de aves rapaces) o la riqueza de especies de la comunidad. Para fines de nuestra propuesta metodológica, se define la propiedad de interés $\mathrm{P}$ como "estructura comunitaria”, entendida como la identidad de las especies que componen la comunidad y sus respectivas abundancias poblacionales relativas.

En la aproximación más utilizada para la estimación de riesgo ecológico este se determina y expresa mediante la razón PEC / PNEC, dando como resultado el coeficiente de riesgo (RQ) (Medina \& Encina 2003). PEC (concentración ambiental predicha, de su sigla en inglés) indica la concentración esperada de un contaminante producto de la operación riesgosa que se está evaluando (e.g. la eventual instalación de una industria). PNEC (concentración sin efecto ecológico predicha) indica la tolerancia al contaminante medida en una especie. Para poder corregir por posibles errores cometidos durante las mediciones efectuadas, por la ignorancia sobre el sistema o especies utilizadas y la variabilidad natural del sistema estudiado, la PNEC es dividida por un Factor de Evaluación, incertidumbre o seguridad (AF) que normalmente asume valores de 10, 50, 100 o 1000, dependiendo del tipo de estudio realizado, el tipo y número de especies utilizadas y la calidad de los datos 
ecotoxicológicos obtenidos (Sprague 1995, Van Leeuwen \& Hermens 1995, USEPA 1998). De esta forma, la fórmula que expresa el riesgo ecológico, medido a través del índice $R Q$ está dada por:

$$
\mathrm{RQ}=\frac{\mathrm{PEC} \cdot \mathrm{AF}}{\mathrm{PNEC}}
$$

Bajo esta aproximación, el cálculo del PNEC se realiza sobre especies de Relevancia ecológica. Sin embargo, aún no se dispone de un protocolo para su identificación ni para la estimación de su valor de relevancia ecológica. Apuntando en dicha dirección, aquí presentamos un marco conceptual formal que permite despejar una expresión de relevancia ecológica en el contexto del concepto de riesgo ecológico. Con este fin es útil formular la siguiente pregunta: ¿de qué depende la generación de efectos ecológicos adversos en una comunidad natural en que una única especie es perturbada por un agente estresante? En otras palabras, ¿cuál es el riesgo ecológico parcial asociado a un factor ambiental sobre una propiedad $\mathrm{P}$ de la comunidad, cuando el factor impacta exclusivamente a una especie focal? En este contexto, $\mathrm{P}$ corresponde a la propiedad que se requiere proteger y debe ser una característica medible de la comunidad focal.

\section{Formalización}

Sean $P, N_{i}, a_{j}$ y $x_{k}$ variables aleatorias donde $P$ representa el parámetro de interés de la comunidad focal, $\mathrm{N}_{\mathrm{i}}$ el tamaño poblacional de la especie $i, a_{j}$ el valor de un rasgo fenotípico $j$ de la especie i y $\mathrm{x}_{\mathrm{k}}$ el valor de un factor ambiental $\mathrm{k}$ que afecta la expresión del rasgo $\mathrm{a}_{\mathrm{j}}$, por ejemplo un agente estresante. Podemos entonces definir las siguientes funciones:

$$
\begin{aligned}
& \mathrm{P}=\mathrm{P}\left(\mathrm{N}_{1}, \ldots, \mathrm{N}_{\mathrm{s}}, \mathrm{L}_{1}, \ldots, \mathrm{L}_{\mathrm{c}}\right) \\
& \mathrm{N}_{\mathrm{i}}=\mathrm{N}_{\mathrm{i}}\left(\mathrm{a}_{1}, \ldots, \mathrm{a}_{\mathrm{m}}\right) \\
& \mathrm{a}_{\mathrm{j}}=\mathrm{a}_{\mathrm{j}}\left(\mathrm{x}_{1}, \ldots, \mathrm{x}_{\mathrm{a}}\right)
\end{aligned}
$$

$$
\begin{aligned}
& \Omega_{\mathrm{i}}=\mathrm{E}\left(\Delta_{\mathrm{i}} \mathrm{P}\right) \\
& \Gamma_{\mathrm{ji}}=\mathrm{E}\left(\Delta_{\mathrm{j}} \mathrm{N}_{\mathrm{i}}\right) \\
& \Phi_{\mathrm{kj}}=\mathrm{E}\left(\Delta_{\mathrm{k}} \mathrm{a}_{\mathrm{j}}\right)
\end{aligned}
$$

donde $\mathrm{L}_{\mathrm{i}}$ representa la magnitud de los enlaces (interacciones ecológicas) entre las especies y donde $1=1, \ldots, \mathrm{c} ; \mathrm{i}=1, \ldots, \mathrm{s} ; \mathrm{j}=1, \ldots, \mathrm{m}_{\mathrm{i}} ; \mathrm{k}=$ $1, \ldots, n$. El parámetro c representa la riqueza de interacciones de la comunidad focal, s es la riqueza de especies o número de taxa en la comunidad focal, $\mathrm{m}_{\mathrm{i}}$ es el número de rasgos fenotípicos que afectan el tamaño poblacional de la especie i, y $\mathrm{n}$ el número de factores ambientales que afectan el valor del rasgo $j$. El operador $\Delta_{\mathrm{y}}$ es definido como sigue. Sea la función $\mathrm{X}=\mathrm{X}\left(\mathrm{z}_{1}, \ldots, \mathrm{z}_{\mathrm{r}}\right)$ con $\mathrm{y}=1, \ldots, \mathrm{r}$ Entonces, definimos la diferencia parcial $\Delta_{\mathrm{y}} \mathrm{X}=\mathrm{X}\left(\mathrm{z}_{1}, \ldots, \mathrm{z}_{\mathrm{y}}+\mathrm{h}, \ldots, \mathrm{z}_{\mathrm{r}}\right)-\mathrm{X}\left(\mathrm{z}_{1}, \ldots, \mathrm{z}_{\mathrm{y}}, \ldots, \mathrm{z}_{\mathrm{r}}\right)$ siendo $h$ un incremento finito de la variable $Z_{\mathrm{y}}$ (cf. Courant et al. 1967). Cuando los límites de evaluación sean explícitos se utilizará la notación $\left.\Delta_{\mathrm{y}} \mathrm{X}\right|_{\mathrm{z}_{\mathrm{y}}} ^{\mathrm{z}_{\mathrm{y}}+\mathrm{h}}$ para representar la misma expresión. La expresión $\mathrm{E}(\mathrm{w})$ representa la esperanza de la variable w. El valor $\Omega_{\mathrm{i}}$ representa la "relevancia ecológica" de la especie i, una expresión de la sensibilidad del parámetro comunitario $\mathrm{P}$ a cambios en el tamaño poblacional de la especie i (ver más adelante), $\Gamma_{\mathrm{ji}}$ representa la sensibilidad del tamaño poblacional de $i$ al cambio en el rasgo $a_{\mathrm{j}}$ y $\Phi_{\mathrm{kj}}$ la variación del valor de un rasgo de la especie i ("endpoint") en respuesta a la magnitud o dosis del estresante, es decir, la sensibilidad del rasgo $a_{j}$ al estresante k. Dentro de los supuestos implícitos en estas relaciones, suponemos que la topología de la red es invariante a la exposición al estresante, y que la magnitud de los enlaces (comúnmente medidos como efectos per cápita, Wootton \& Emmerson 2005) es también invariante o bien no juega un rol determinante en la relación entre la abundancia de las especies y la propiedad comunitaria de interés.

Dado que en la práctica (a) es difícil hacer suficientes réplicas para estimar las esperanzas de las variables desde distribuciones de probabilidad de diferencias y (b) para efectos de evaluación de riesgo ecológico es relevante estimar los efectos potenciales de la concentración esperada de 
un estresante (PEC), podemos aplicar las siguientes expresiones para estimar $\Phi_{\mathrm{kj}}, \Gamma_{\mathrm{ji}}$ y $\Omega_{\mathrm{i}}$ respectivamente:

$$
\begin{aligned}
& \varphi_{\mathrm{kj}}=\left.\Delta_{\mathrm{k}} \mathrm{a}_{\mathrm{j}}\left(\mathrm{x}_{\mathrm{k}}\right)\right|_{\mathrm{x}_{\mathrm{k}}=\mathrm{C}} ^{\mathrm{x}_{\mathrm{k}}=\mathrm{PEC}} \\
& \gamma_{\mathrm{ji}}=\Delta_{\mathrm{j}} \mathrm{N}_{\mathrm{i}}\left(\mathrm{a}_{\mathrm{j}}\right) \mid \begin{array}{l}
\mathrm{a}_{\mathrm{j}}\left(\mathrm{x}_{\mathrm{k}}=\mathrm{PEC}\right) \\
\mathrm{a}_{\mathrm{j}}\left(\mathrm{x}_{\mathrm{k}}=\mathrm{C}\right)
\end{array} \\
& \omega_{\mathrm{i}}=\Delta_{\mathrm{i}} \mathrm{P}\left(\mathrm{N}_{\mathrm{i}}\right) \quad \begin{array}{l}
\mathrm{N}_{\mathrm{i}}\left(\mathrm{a}_{\mathrm{j}}\left(\mathrm{x}_{\mathrm{k}}=\mathrm{PEC}\right)\right) \\
\mathrm{N}_{\mathrm{i}}\left(\mathrm{a}_{\mathrm{j}}\left(\mathrm{x}_{\mathrm{k}}=\mathrm{C}\right)\right)
\end{array}
\end{aligned}
$$

donde PEC es la concentración ambiental esperada del estresante $\mathrm{k}$ y $\mathrm{C}$ es la concentración control o referencial del mismo. El valor de PEC es una salida del proceso de caracterización de la exposición en ERE (Medina et al. 2011). En la fase de caracterización de los efectos en ERE, puede estimarse $\varphi_{\mathrm{kj}}$, indicador íntimamente relacionado con PNEC (definido más arriba), a partir de curvas dosis-respuesta provenientes de pruebas de laboratorio estandarizadas (bioensayos). La extrapolación de efectos individuales a niveles superiores de organización está contenida en la estimación de $\gamma_{\mathrm{ji}}$ y $\omega_{\mathrm{i}}$, que comúnmente no es considerado explícitamente. Si bien estos parámetros pueden estimarse a través de experimentos, la dificultad de manipulación y registro de variables respuesta hace deseable una aproximación teórica.

Finalmente, podemos definir

$$
\Psi_{\mathrm{ijk}}=\Omega_{\mathrm{i}} \mid \begin{aligned}
& \mathrm{x}_{\mathrm{k}}=\mathrm{PEC} \\
& \mathrm{x}_{\mathrm{k}}=\mathrm{C}
\end{aligned}
$$

como el "riesgo ecológico parcial" de la comunidad cuando es alterado un rasgo $\mathrm{j}$ de la especie i. Este indicador predice el impacto que ejerce la concentración esperada del contaminante $\mathrm{k}$ sobre el valor del parámetro comunitario $\mathrm{P}$, a través de su efecto sobre la especie $\mathrm{i}$, en relación al valor del parámetro comunitario en la concentración control de contaminante. Este impacto está mediado por el efecto del contaminante sobre el valor del rasgo fenotípico $\mathrm{j}$ considerado, el cual afecta el tamaño poblacional de la especie, el que finalmente determina el parámetro comunitario. El "riesgo ecológico total" de la comunidad a la exposición del contaminante estaría dado por una función del tipo

$$
\Psi_{\mathrm{k}}=f(\mathrm{M})
$$

que integre los efectos esperados sobre las $\mathrm{s}$ especies de la comunidad, con

$$
\mathrm{M}=\left[\begin{array}{ccc}
\Psi_{11 \mathrm{k}} & \cdots & \Psi_{1 \mathrm{mk}} \\
\vdots & \ddots & \vdots \\
\Psi_{\mathrm{s} 1 \mathrm{k}} & \cdots & \Psi_{\mathrm{smk}}
\end{array}\right]
$$

\section{Relevancia ecológica}

Entenderemos como una especie ecológicamente relevante (EER) aquella que al ser perturbada por un estresante genera cambios relativamente drásticos en la propiedad comunitaria de interés, relativo a lo que provocaría una perturbación de igual magnitud ejercida sobre otras especies de la comunidad (DFO 2007). Desde este concepto, se define relevancia ecológica de una especie i, $\Omega_{\mathrm{i}}$, como la magnitud de cambio en una propiedad comunitaria $\mathrm{P}$ en función del cambio en la abundancia de la especie i, $\mathrm{N}_{\mathrm{i}}$. La propiedad P, por otro lado, corresponde a aquella característica de la comunidad que representa el objetivo de manejo. Así, cada especie de la comunidad tendría un valor asociado de relevancia ecológica. Las especies que presenten mayores valores de $\Omega_{\mathrm{i}}$, es decir, aquellas en que un eventual cambio en su abundancia afectaría en mayor magnitud a $\mathrm{P}$, serían las candidatas para aplicárseles pruebas ecotoxicológicas a fin de evaluar los efectos del estresante $\mathrm{k}$ sobre atributos de estas y así estimar el riesgo ecológico de la comunidad, dada una exposición a k.

\section{IDENTIFICACIÓN DE ESPECIES ECOLÓGICAMENTE RELEVANTES}

El método de identificación de EER dependerá de dos condiciones básicas: (a) el nivel de conocimiento del sistema focal (NCSF), es decir de la calidad de la información acerca de la estructura de la comunidad focal y (b) de la 
capacidad del investigador (tiempo, recursos) para manipular experimentalmente el sistema (CMS). En general, para efectos de ERE y otras medidas tendientes a la gestión ambiental en la Región, la posibilidad de ejecutar experimentos manipulativos es nula o severamente limitada (i.e., CMS bajo). Esta restricción impone buscar métodos de selección de EER en base a la información disponible. Por otro lado, podemos clasificar el NCSF de un ecosistema o comunidad en tres grandes categorías. Bajo, cuando solo se dispone de un listado de las especies más abundantes que componen la comunidad y de un conocimiento general de la biología de ellas; medio, cuando se conoce (a) un buen número de las especies más importantes numéricamente y más conspicuas de la comunidad, y (b) las relaciones tróficas entre grupos funcionales que incluyen a las especies conocidas de la comunidad; alto, cuando se conoce la composición comunitaria mayoritariamente a nivel de especies, así como sus abundancias y la dirección y fuerza de sus interacciones ecológicas (i.e., una red ecológica con alta resolución). En la actualidad, el NCSF para comunidades acuáticas chilenas se encuentra en bajo o medio para la totalidad de las cuencas hidrográficas. Esta situación no se prevé que mejore sustancialmente en el siguiente quinquenio, por lo cual este estudio se refiere a métodos aplicables solo a sistemas con CMS bajo y NCSF bajo y medio.

\section{Criterios cualitativos}

Corresponden a criterios orientados a estimar cualitativamente la importancia de cada especie para el mantenimiento de la estructura de la comunidad. Estos criterios tienen dos aplicaciones: (a) cuando el NCSF es bajo y (b) como segundo paso tras haber seleccionado grupos funcionales ecológicamente relevantes en sistemas con NCSF medio (ver más adelante). En base nuestra experiencia y al conocimiento disponible, se propone el siguiente conjunto de criterios cualitativos para la selección de EER. (i) Dominancia numérica en biomasa: especies con mayor biomasa presentan mayor $\Omega$ que especies raras debido a que participan con mayor peso en los flujos de materia y energía dentro de las tramas tróficas. (ii) Distribución en el sistema: especies ampliamente distribuidas ejercen influencias directas sobre una mayor fracción del ecosistema y por tanto presentan mayor $\Omega$. (iii) Persistencia estacional: especies que están presentes (en estado de vida libre) una mayor proporción del ciclo estacional poseen mayor $\Omega$ debido a que influyen en el sistema de manera temporalmente sostenida. (iv) Tamaño corporal: especies con mayor tamaño corporal están asociadas a mayores $\Omega$ debido a que presentan mayores tasas metabólicas y de consumo de recurso (Brown et al. 2004) $y$, consecuentemente, generan fuerzas de interacción de mayor magnitud (Berlow et al. 2009). (v) Conocimiento biológico: El juicio experto acerca de la biología de las especies del sistema de interés y el conocimiento acerca del funcionamiento de este podrá revelar propiedades biológicas especiales (e.g. modificadores de hábitat, facilitadores, antagonistas directos, etc.) que permita elevar el valor relativo de $\Omega$ respecto de otras especies presentes. (vi) Representación de al menos tres niveles tróficos (solo para aplicación (i)): es importante seleccionar especies que formen parte de diferentes niveles tróficos en la comunidad debido a que especies de nivel trófico basal determinan la regulación ascendente (bottom-up) de la diversidad, que las especies de mayor nivel trófico determinan la regulación descendente (top-down) y las especies intermedias (e.g., herbívoros) son importantes como transmisores de biomasa en la trama trófica.

Uso de criterios cualitativos en sistemas con bajo nivel de conocimiento

En la Tabla 1 se presentan niveles o posiciones tróficas usualmente definidos para comunidades acuáticas, agrupados en cuatro categorías tróficas. Se propone que como primer paso, y basados en la información disponible del sistema de estudio, se defina el número de categorías tróficas dentro de las cuales se seleccionarán las especies ecológicamente relevantes. Sugerimos que este número sea al menos tres.

Para cada categoría trófica a considerar en el análisis, se calcula un valor de relevancia ecológica parcial para cada una de sus especies constituyentes conocidas, en base a los cinco criterios cualitativos ya mencionados. Una propuesta simple de cálculo de relevancia 
TABLA 1

Definición de categorías tróficas para sistemas acuáticos con bajo nivel de conocimiento.

\begin{tabular}{lc}
\hline Nivel trófico & Categoría trófica \\
\hline Productor primario (PP) & i \\
Herbívoro (H). Consume a PP & ii \\
Detritívoro (DV) & iii \\
Depredador primario (D1). Consume a H o DV & iv \\
Depredador secundario (D2). Consume a D1 & \\
Depredador terciario (D3). Consume a D2 & \\
\hline
\end{tabular}

ecológica mediante criterios cualitativos se presenta en el Material Complementario, sección A. Así, se obtiene un ranking del valor de relevancia ecológica $(\Omega)$ para cada una de las especies pertenecientes a una categoría trófica. Las especies con mayor relevancia ecológica serán seleccionadas con mayor prioridad para la ejecución de bioensayos que permitan la estimación del efecto dentro del procedimiento ERE.

Uso de criterios cualitativos en sistemas con nivel medio de conocimiento

El proceso de selección de EER para este tipo de sistemas consiste en dos pasos. Primero, mediante índices cuantitativos (ver siguiente sección) se seleccionan los grupos funcionales de mayor relevancia ecológica. Segundo, para cada uno de los grupos funcionales seleccionados, se aplican los criterios cualitativos, que arrojan valores relativos de relevancia ecológica para las especies de cada grupo funcional seleccionado.

\section{ÍNDICES CUANTITATIVOS}

Corresponden a índices que arrojan valores indicativos del nivel de importancia de cada componente (especie, trofo-especie o grupo funcional) para el mantenimiento de la estructura de la comunidad. Para los propósitos de este trabajo, los índices cuantitativos se orientarán hacia la identificación de grupos funcionales (GFs) ecológicamente relevantes, dentro de sistemas con NCSF medio. Los índices cuantitativos pueden agruparse en dos grandes categorías: (a) índices topológicos, los cuales evalúan la importancia de un componente de la red para el mantenimiento de la estructura de la comunidad; (b) índices basados en modelación y/o simulación, los cuales permiten evaluar la importancia de un componente de la red para la dinámica de la comunidad.

\section{Índices topológicos}

Los índices topológicos son aquellos que, para evaluar la importancia de un componente de la comunidad, requieren como información de entrada solo el número de componentes y sus relaciones en el sistema de interés. En Material Complementario sección B se presenta una recopilación de los índices más utilizados en la literatura de redes ecológicas.

Para los propósitos de este estudio se han seleccionado siete de los índices recopilados. Nuestros criterios favorecieron aquellos índices con mayor significancia ecológica, más extensiva utilización actual (validación) en la literatura científica, y más clara interpretabilidad. Los índices seleccionados son: Keystone Index (KI_TOT), Keystone Index bottom-up (KI_BU), Keystone Index TopDown (KI TD), Betweenness Centrality NonDirected (BC_ND), Betweenness Centrality Directed (BC_D), Eigenvector Centrality (EC) y Topological Importance (TIn). Véase el Material Complementario, sección C, para una explicación de estos índices y su cálculo.

\section{Índices basados en modelización y/o simulación}

Son métricas que resultan del análisis o simulación numérica de un modelo matemático 
dinámico. La información de entrada requerida depende de la estructura del modelo. Modelos cuantitativos son representados generalmente como un sistema de ecuaciones diferenciales o en diferencia, y requieren de valores iniciales de variables y de valores de parámetros de sus relaciones funcionales. A la vez, las relaciones funcionales (tasas de crecimiento $\mathrm{y}$ regulación, tasas de depredación, etc.) deben ser definidas a priori. Cambios en condiciones iniciales, valores de parámetros y estructura de relaciones funcionales a menudo ejercen un impacto importante sobre los resultados del modelo. Esto impone que los modelos (o simulaciones) cuantitativas solo puedan llevarse a cabo con un NCSF alto. Existe una alternativa para aplicar índices basados en modelización para nuestros sistemas de interés (i.e. NCSF medio). Esta corresponde a un conjunto de herramientas basadas en teoría de sistemas dinámicos conocida como Análisis Cualitativo de Sistemas Complejos, o análisis de ciclos (Puccia \& Levins 1985, Dambacher et al. 2003a, b, Dambacher \& Ramos-Jiliberto 2007). Esta aproximación permite obtener información valiosa acerca de sistemas parcialmente especificados (solo por sus componentes y relaciones), sin necesidad de incorporar valores de parámetros, abundancias iniciales o relaciones funcionales específicas (Wootton \& Emmerson 2005). El supuesto más fuerte de este tipo de modelo es que el sistema debe encontrarse en un equilibrio localmente estable, lo cual en rigor limita fuertemente su aplicación a sistemas fuera del equilibrio y particularmente a sistemas con relaciones no lineales marcadas (Justus 2006). Sin embargo, difícilmente se podrá establecer si la comunidad focal se encuentra en equilibrio cuando se carece de información detallada de las abundancias poblacionales a través del tiempo. Entonces, consideramos útil incorporar el análisis de ciclos, el cual nos informa qué cambios ocurrirían si el sistema estuviese en equilibrio y es sometido a una pequeña perturbación de presión (Bender et al. 1984).

Aquí utilizamos herramientas de modelamiento cualitativo para estimar un índice de relevancia ecológica (ADJ_NEG) basado en el número estimado de nodos de la comunidad que se predice disminuyan su abundancia al perturbar al nodo focal. Véase el Material Complementario, sección
$\mathrm{D}$, para una explicación de este índice y su cálculo. En el caso que exista información suficiente que indique que el sistema no se encuentra en equilibrio y las predicciones de este análisis se desvían notablemente de las predicciones obtenidas desde los otros índices, sería concebible eliminar el análisis de ciclos del procedimiento para identificar EERs.

\section{PROCEDIMIENTO PARA EL USO DE ÍNDICES CUAN- TITATIVOS}

Para la integración de las diferentes métricas aplicadas para la selección de EER, se propone un procedimiento en cuatro pasos.

Paso 1: Cálculo del valor de cada índice para cada nodo (GF). Se definen tres tipos de índices cuantitativos. Tipo I: KI_TD, KI_BU y BC_D. Éstos tienden a otorgar mayor valor a nodos de niveles tróficos altos, bajos e intermedios respectivamente. Tipo II: EC, BC_ND, TI3_ ND y KI_TOT. Son índices de importancia topológica global, que no tienden a privilegiar niveles tróficos particulares. Los tres primeros son índices para grafos no dirigidos y el último es para grafos dirigidos. Tipo III: ADJ_NEG. Este índice proviene de modelación cualitativa y considera la dinámica del sistema. El grafo (comunidad) debe tener vínculos entre nodos con dirección y signo de las interacciones que representan.

Paso 2: En base al valor que arroja cada índice, se obtiene el ranking $\mathrm{X}_{\mathrm{i}}$ de cada i-ésimo nodo (para cada índice). Por ejemplo, para una comunidad con 4 nodos y con valores de KI_TD $=[0,3,3,4]$, se obtiene el ranking $[4,2,2,1]$.

Paso 3: De los 8 índices originales se obtienen 5 índices transformados, según se detalla a continuación para cada tipo de índice. Tipo I: Se transforman los valores de ranking para los índices de este tipo. Para cada índice $X$, el valor por nodo $X_{i}$ se transforma a $T_{i}$ según:

$$
T_{i}\left\{\begin{array}{cc}
1 & \text { si } X_{i}=1 \\
\operatorname{round}\left(n^{-1} \sum_{j=1}^{n} X_{j}\right) & \text { si } X_{i}>1
\end{array}\right.
$$

donde el operador round(x) indica redondeo al entero, $n$ es el número de nodos de la comunidad. Así, el ranking transformado 
puede asumir solo dos valores posibles: 1 o el promedio del valor $\mathrm{X}_{\mathrm{i}}$ de los nodos. Este paso tiene por objeto el remover el castigo a los nodos que se ubican en niveles tróficos diferentes al favorecido por el índice. Tipo II: Se promedian, para cada nodo, los valores de ranking $\mathrm{X}_{\mathrm{i}}$ sobre los cuatro índices que pertenecen al Tipo II. Esto tiene como propósito integrar los valores de los índices Tipo II en un único valor. Tipo III: Se mantiene el valor para ADJ_NEG.

Paso 4: se promedian, para cada nodo, los valores de los cinco índices transformados $T_{\mathrm{i}}$. Este valor promedio se denomina $\mathrm{R}_{\mathrm{i}}$, para cada i-ésimo nodo de la comunidad. Se registran los valores máximo $\left(R_{\max }\right)$ y mínimo $\left(R_{\min }\right)$ entre $\operatorname{los} \mathrm{R}_{\mathrm{i}}$ de la comunidad. Finalmente, se calcula

$$
\Omega_{\mathrm{i}}=\frac{\mathrm{R}_{\max }-\mathrm{R}_{1}}{\mathrm{R}_{\max }-\mathrm{R}_{\min }}
$$

donde $\Omega_{\mathrm{i}}$ corresponde a la relevancia ecológica normalizada (entre 0 y 1 ) del i-ésimo nodo de la comunidad. Para la selección de GFs (nodos) ecológicamente relevantes, se incluye la cantidad necesaria de nodos con mayor valor de $\Omega_{\mathrm{i}}$, hasta incluir al menos tres categorías tróficas diferentes, según se definieron en Tabla 1. En el Material Complementario, sección A, se muestra un ejemplo del cálculo paso a paso para la obtención de $\Omega_{\mathrm{i}}$ en una comunidad estructurada en grupos funcionales tomada de Ramos-Jiliberto et al. (2012). En el Material Complementario, sección $\mathrm{G}$ se muestra un código MATLAB desarrollado para este trabajo, que permite obtener directamente el valor de $\Omega_{\mathrm{i}}$, a partir de los valores de los 8 índices descritos más arriba.

\section{ESQUEMA GENERAL PARA LA IDENTIFICACIÓN} DE EER

En la Fig. 2 se resume el procedimiento desarrollado para la identificación de EER. Para sistemas con bajo NCSF se utilizan criterios cualitativos. Para sistemas con nivel medio de conocimiento se utilizan índices cuantitativos para la selección de GFs ecológicamente relevantes. Luego, se determinan las EER dentro de los GFs seleccionados por medio del uso de criterios cualitativos. Como salida, se obtienen especies representativas de tres o más categorías tróficas como candidatas para la determinación de los efectos en una ERE. Del conjunto de especies candidatas, no todas podrían ser apropiadas para la ejecución de bioensayos. Así, finalmente operan criterios adicionales para la decisión de qué especies utilizar para la determinación de los efectos. Dos criterios son fundamentales para esta decisión: (1) el costo de aclimatar y mantener a los organismos en condiciones experimentales apropiadas, (2) la sensibilidad esperada de los organismos a los estresores de interés. En resumen, en la ejecución de una ERE se determinarán los efectos de los estresores en especies con mayor relevancia ecológica, perteneciente al menos a tres categorías tróficas, que sean sujetos factibles de experimentación y cuya sensibilidad esperada sea alta.

\section{Direcciones futuras}

Existen dos limitaciones mayores al avance de las proyecciones de la dinámica de comunidades naturales sujetas a perturbaciones. La primera la constituye el estado de desarrollo de las herramientas conceptuales y analíticas para formular predicciones. En este campo el avance de la teoría ecológica y en particular de la ecología de redes es auspicioso. La segunda descansa en la calidad de la información empírica disponible, particularmente el conocimiento de la diversidad biológica local en todos sus niveles de organización. Mejores datos de abundancia, distribución, dinámica, arquitectura comunitaria, magnitudes de interacción, serán definitivamente útiles considerando las herramientas analíticas disponibles y las que activamente se desarrollan en las ciencias ecológicas actualmente. E1 trabajo de campo cuidadoso, unido a un sólido conocimiento teórico en ecología posibilita el desarrollo de mejores herramientas de gestión. El método presentado en esta propuesta descansa, necesariamente, en el supuesto que el sistema está correctamente representado. Desviaciones importantes de la estructura real de la comunidad focal en sistemas con NCSF medio puede conducir a identificaciones erróneas de las especies más relevantes ecológicamente. Sin embargo, no es extremadamente difícil obtener una adecuada 


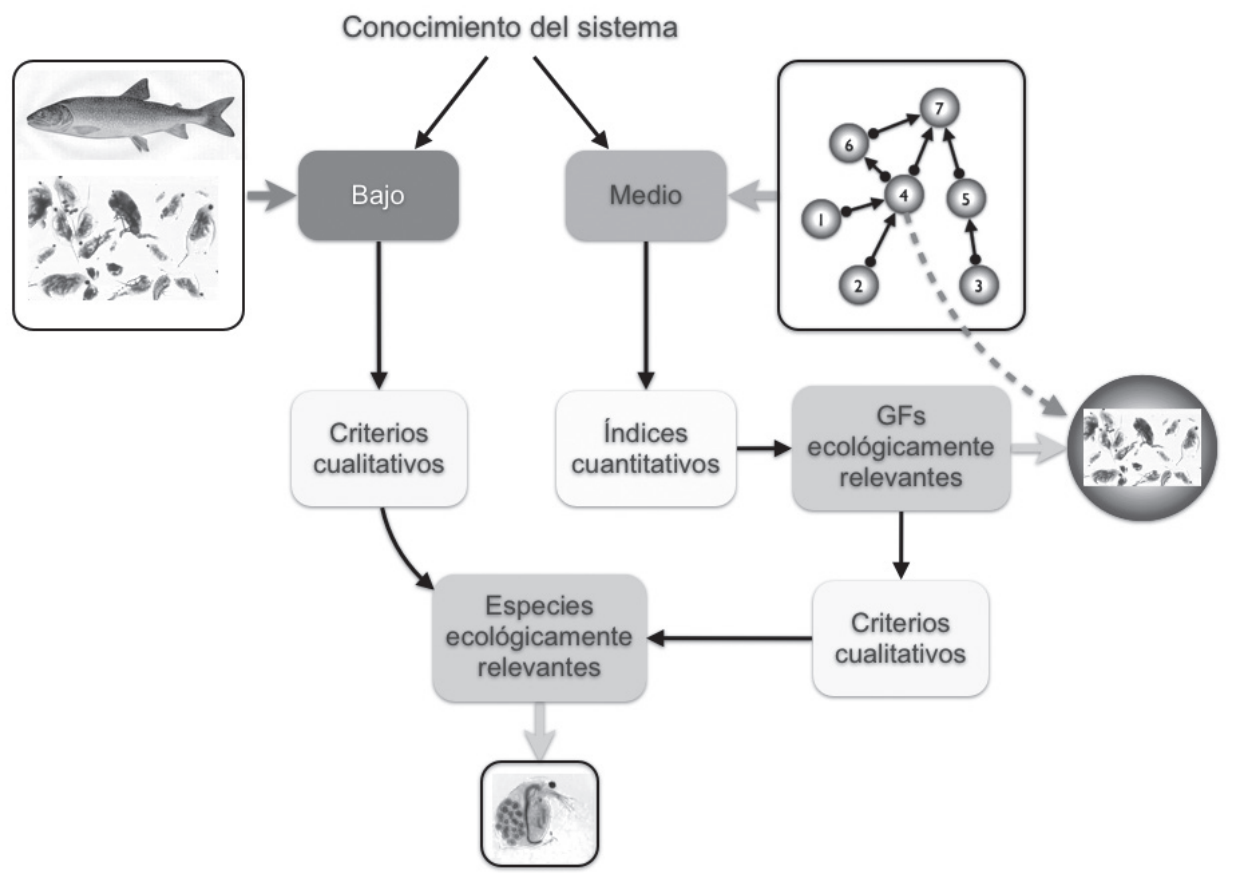

Fig. 2: Esquema general del procedimiento propuesto para la identificación de EER.

General outline of the proposed procedure for the identification of EER.

representación de la estructura comunitaria con resolución taxonómica de grupos tróficos (NCSF medio). Por un lado, habría que omitir el registro de todas las especies de un grupo trófico para omitir el nodo correspondiente. Por otro lado, las relaciones tróficas entre grupos funcionales están establecidas en la literatura y no es absolutamente necesario realizar observaciones ad-hoc. Sin embargo, nuestra capacidad de gestión de los ecosistemas acuáticos se beneficiará considerablemente del desarrollo futuro de descripciones comunitarias de alta resolución, a nivel de especies o trofoespecies. Este desarrollo, sin embargo, requiere de una cantidad de información y experimentación que aún estamos lejos de disponer en América Latina, salvo para casos de estudio particulares. Esperamos que esta contribución sea útil para la evaluación de riesgo ecológico en ecosistemas acuáticos parcialmente descritos y sirva de motivación para avanzar en el estudio detallado de nuestros sistemas naturales. Esta propuesta constituye un avance desarrollado con atención, pero éste debe ser discutido por la comunidad científica y validado -en su estado actual o mejorado- mediante su aplicación experimental en sistemas reales.

\section{MATERIAL COMPLEMENTARIO}

Los códigos, índices y cálculos aplicados en este artículo están disponibles como Material Complementario en: http://rchn.biologiachile. cl/pdfs/2013/1/MC_Ramos-Jiliberto_et_ al_2013.pdf

AGRADECIMIENTOS: Los autores agradecen al Ministerio de Medio Ambiente del Gobierno de Chile por facilitar el uso de la información contenida en este trabajo. Financiamiento parcial de proyectos FONDECYT 1090132 y 1120958.

\section{LITERATURA CITADA}

BENDER EA, TJ CASE \& ME GILPIN (1984) Perturbation experiments in community ecology: theory and practice. Ecology 65: 1-13.

BERLOW EL, JA DUNNE, ND MARTINEZ, PB STARK, RJ WILLIAMS \& U BROSE (2009) Simple prediction of interaction strengths in complex food webs. Proceedings of the National Academy of Sciences USA 106: 187-191. 
BROWN JH, JF GILLOOLY, AP ALLEN, VM SAVAGE \& GB WEST (2004) Toward a metabolic theory of ecology. Ecology 85:1771-1789.

COURANT R, FRIEDRICHS KO \& H LEWY (1967) On the partial difference equations of mathematical physics, IBM Journal 11: 215-235.

DAMBACHER JM, HW LI \& PA ROSSIGNOL (2003a) Qualitative predictions in model ecosystems. Ecological Modelling 161: 79-93.

DAMBACHER JM, H LUH, HW LI \& PA ROSSIGNOL (2003b) Qualitative stability and ambiguity in model ecosystems. The American Naturalist 161: 876-888.

DAMBACHER JM \& R RAMOS-JILIBERTO (2007) Understanding and predicting effects of modified interactions through a qualitative analysis of community structure. The Quarterly Review of Biology 82: 227-250.

DFO (2007). Identification of ecologically significant species and community properties. Department of Fisheries and Oceans Canada. Science Advisory Report 2006/041.

ENCINA F \& O DÍAZ (2001) Contaminación, estimación del riesgo ecológico y protección asociado a algas bentónicas marinas. En: Alvear K \& T Antezana (eds) Sustentabilidad de la biodiversidad: 336-357. Universidad de Concepción. Concepción, Chile.

JUSTUS J (2006) Loop analysis and qualitative modeling: limitations and merits. Biology and Philosophy 21: 647-666.

MEDINA M \& F ENCINA (2003) Incorporación de la Evaluación de Riesgo Ecológico en el Sistema de Evaluación de Impacto Ambiental para ecosistemas acuáticos en Chile. Revista Ambiente y Desarrollo (Chile) 19: 19-26.

MEDINA MH, P IBIETA, L MARTÍNEZ \& A MEDINA (2011) Evaluación comparada sobre legislación internacional aplicada y metodologías de Evaluaciones de Riesgo para incorporar en la elaboración de políticas de prevención y contaminación y regulaciones ambientales. Informe Técnico elaborado por AVS Chile para el Ministerio de Medio Ambiente, Santiago, Chile, 139 pp.

PUCCIA CJ \& R LEVINS (1985) Qualitative Modeling of Complex Systems. An Introduction to Loop Analysis and Time Averaging. Harvard University Press, Cambridge, USA

RAMOS-JILIBERTO R, L GARAY-NARVÁEZ \& MH MEDINA (2012) Retrospective qualitative analysis of ecological networks under environmental perturbation: a copper-polluted intertidal community as a case study. Ecotoxicology 21: 234-243.

RAND G, P WELLS \& L McCARTY (1995) Introduction to ecological risk assessment. En: Rand G. (Ed.) Fundamentals of aquatic toxicology. Effects, environmental fate and risk assessment: 1125 . Taylor \& Francis, London.

SIMONETTI JA (2011) Conservation biology in Chile: Are we fulfilling our social contract? Revista Chilena de Historia Natural 84: 161-170.

SPRAGUE J (1995) Factors that modify toxicity. En: Rand G (ed) Fundamentals of aquatic toxicology. Effects, environmental fate and risk assessment: 1012-1213. Second edition, Taylor and Francis, London.

USEPA (1992) Framework for ecological risk assessment. Washington, DC: Risk Assessment Forum, U.S. Environmental Protection Agency. EPA/630/R-92/001.

USEPA (1998) Guidelines for Ecological Risk Assessment. Washington, DC: Risk Assessment Forum, U.S. Environmental Protection Agency. EPA/630/R-95/002F.

VAN LEEUWEN C \& J HERMENS (1995) Risk assessment of chemicals: an introduction. Kluwer Academic Publishers, Netherlands.

WOOTTON JT \& M EMMERSON (2005) Measurement of interaction strength in nature. Annual Review of Ecology, Evolution and Systematics 36: 419-44. 
